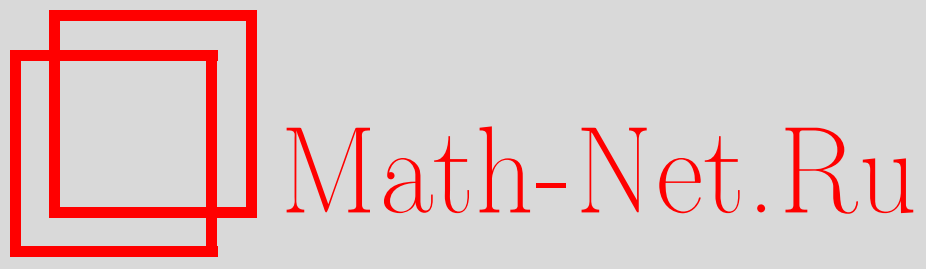

А. Э. Бирюк, Спектральные свойства решений уравнения Бюргерса с малой диссипацией, Функи. анализ и его прил., 2001, том 35, выпуск 1, 1-15

DOI: https://doi.org/10.4213/faa227

Использование Общероссийского математического портала MathNet.Ru подразумевает, что вы прочитали и согласны с пользовательским соглашением

http://www . mathnet.ru/rus/agreement

Параметры загрузки:

IP: 54.162 .27 .143

26 апреля 2023 г., 17:47:00

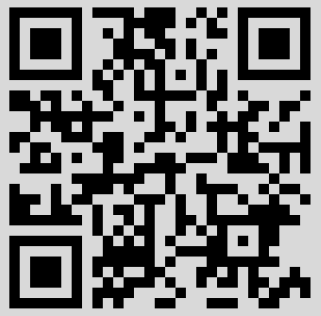


УДК 517.9

\title{
Спектральные свойства решений уравнения Бюргерса с малой диссипацией
}

\author{
(c) 2001. А. Э. БИРЮК
}

\section{§1. Введение}

В этой работе мы рассматриваем задачу Коши для одномерного $(\operatorname{dim} x=1)$ параболического уравнения типа Бюргерса

$$
\frac{\partial}{\partial t} u+\frac{\partial}{\partial x} f(u)=\delta u_{x x}
$$

с начальными данными

$$
u(0, x)=u_{0}(x),
$$

подчиненного периодическим краевым условиям

$$
u(t, x \pm \ell)=u(t, x) .
$$

Здесь $\delta$ - малый $(<1)$ положительный параметр, начальные данные $-C^{\infty}$-гладкая и $\ell$-периодическая функция $u_{0}(\cdot)$. Функция $f: \mathbb{R} \rightarrow \mathbb{R}$ бесконечно гладкая и равномерно выпуклая $\left(f^{\prime \prime} \geqslant \sigma>0\right)$.

Из классической теории параболических уравнений (см., например, [1]) известно, в частности, что задача (1.1)-(1.3) при каждом $\delta>0$ имеет единственное решение $u=u^{\delta}(t, x)$, которое является $C^{\infty}$-гладким. Кроме того, известно, что при $\delta \rightarrow+0$ решение задачи (1.1)-(1.3) сходится к так называемому (слабому) энтропийному решению квазилинейного уравнения

$$
\frac{\partial u}{\partial t}+\frac{\partial f(u)}{\partial x}=0
$$

(См. [2], где этот вопрос изучен при самых общих предположениях.) Более того, в [3] показано, что скорость сходимости в $L_{1}(\mathbb{R})$ не ниже Const $\sqrt{\delta}$. По поводу нашего случая см. дополнение.

Отметим, что уравнение $\left(1.1^{\prime}\right)$, вообще говоря, не имеет даже непрерывных решений; поэтому рассматривают слабые решения в смысле соответствующего интегрального тождества. Однако в этом классе происходит потеря единственности. Условие энтропии производит выбор из множества решений одного, которое оказывается наиболее естественным (с точки зрения физики).

Мы будем изучать поведение при $\delta \rightarrow 0$ соболевских $H^{m}$-полунорм

$$
\|u\|_{m}^{2}=\int\left|\frac{\partial^{m} u}{\partial x^{m}}\right|^{2} d x
$$


Всюду в этой работе под $\int \ldots d x$ (интеграл без пределов) понимается $\int_{0}^{\ell} \ldots d x=$ $\int_{x_{0}}^{x_{0}+\ell} \ldots d x-$ интеграл по периоду или, что то же самое, интеграл по окружности $S=\mathbb{R} / \ell \mathbb{Z}$. Приставку «полу»в слове «полунорма» будем для краткости опускать.

Соболевская $H^{m}$-норма является функцией от времени $t$, и нас будет интересовать усреднение

$$
\left\langle\|u\|_{m}^{2}\right\rangle_{[0, T]}=\frac{1}{T} \int_{0}^{T} \int\left|\frac{\partial^{m} u}{\partial x^{m}}\right|^{2} d x d t .
$$

Мы получим, что при $m \geqslant 1$ для достаточно малых $\delta$ и при всех $t$ имеет место неравенство

$$
\|u\|_{m} \leqslant C_{m}\left(\frac{1}{\delta}\right)^{m-1 / 2},
$$

в то время как для некоторого $T$, определенного начальными данными задачи (1.1)-(1.3) (см. формулу (3.2) ниже) имеет место противоположное неравенство

$$
\left\langle\|u\|_{m}^{2}\right\rangle_{[0, T]}^{1 / 2} \geqslant c_{m}\left(\frac{1}{\delta}\right)^{m-1 / 2} .
$$

Здесь константы $c_{m}$ и $C_{m}$ не зависят от $\delta$ и могут быть явно описаны в терминах $C^{k}$-норм $(k \leqslant m+1)$ начальных данных задачи (1.1)-(1.3).

Соболевские $H^{m}$-нормы можно простым образом выразить через коэффициенты Фурье:

$$
\|u\|_{m}^{2}=\left(\frac{2 \pi}{\ell}\right)^{2 m} \sum_{s=-\infty}^{+\infty} s^{2 m}\left|\hat{u}_{s}\right|^{2}, \quad \text { где } \hat{u}_{s}=\frac{1}{\sqrt{\ell}} \int u(x) e^{-2 \pi i s x / \ell} d x .
$$

Эта связь позволяет получить из (1.4) и (1.5) некоторую информацию о поведении коэффициентов Фурье решения при $\delta \rightarrow+0$. А именно, выбрав произвольное $\theta>0$, мы обозначим через $\hat{A}_{s, \theta}^{2}$ среднее значение величин $\left|\hat{u}_{k}\right|^{2}(t)$ при $t \in[0 ; T]$ и при $\delta^{-s+\theta}<|k| \leqslant \delta^{-s-\theta}$, т. е.

$$
\hat{A}_{s, \theta}^{2}=\frac{1}{T} \int_{0}^{T} \frac{\sum_{\delta^{-s+\theta}<|k| \leqslant \delta^{-s-\theta}}\left|\hat{u}_{k}\right|^{2}(t)}{\sum_{\delta^{-s+\theta}<|k| \leqslant \delta^{-s-\theta}} 1} d t,
$$

где $s>0$. Тогда при достаточно малых $\delta$ величины $\hat{A}_{s, \theta}^{2}$ удовлетворяют следующим оценкам:

$$
\begin{array}{ll}
\text { при } s>0 & \hat{A}_{s, \theta}^{2}<\frac{\ell}{\pi^{2}}\left|\frac{d u_{0}}{d x}\right|_{L_{1}}^{2} \delta^{2 s}, \\
\text { при } s>1+\theta & \hat{A}_{s, \theta}^{2}<\left(\frac{\ell}{2 \pi}\right)^{2 m} C_{m}^{2} \delta^{2+2 m(s-1-\theta)} \quad \forall m>0, \\
\text { при } s=1 & \hat{A}_{1, \theta}^{2}>\left(\frac{\ell}{2 \pi}\right)^{2} \frac{c_{1}^{2}}{4} \delta^{2+2 \theta} .
\end{array}
$$

Установление оценок (1.4)-(1.8) для задачи (1.1)-(1.3) и является главной целью настоящей работы.

Отметим, что соотношения (1.6)-(1.8) тесно связаны с так называемыми колмогоровскими асимптотиками для свободно затухающей турбулентности (т.е. 
турбулентности без внешней силы), описываемой уравнением Бюргерса (1.1). В частности, из них следует, что колмогоровский диссипационный масштаб решений этого уравнения равен $\delta$, а показатель их спектрального закона равен двум (см. [8], ср. $[9,10])$.

В дальнейшем нам потребуются интерполяционные неравенства для норм Соболева и для $C^{k}$-норм:

$$
\begin{aligned}
\|u\|_{k} & \leqslant\|u\|_{0}^{1-k / m}\|u\|_{m}^{k / m} & & (0 \leqslant k \leqslant m), \\
|u|_{k} & \leqslant C_{m k}|u|_{0}^{1-k / m}|u|_{m}^{k / m} & & (0 \leqslant k \leqslant m) .
\end{aligned}
$$

Здесь $|u|_{k}=\max _{x \in S}\left|d^{k} u / d x^{k}\right|$. Доказательство неравенства (1.9) настолько просто, что мы его приведем:

$$
\begin{aligned}
& \left(\frac{\ell}{2 \pi}\right)^{2 k}\|u\|_{k}^{2}=\sum\left(\left|\hat{u}_{s}\right|^{2}\right)^{(m-k) / m}\left(\left|\hat{u}_{s}\right|^{2} s^{2 m}\right)^{k / m} \\
& \leqslant\left(\sum\left|\hat{u}_{s}\right|^{2}\right)^{(m-k) / m}\left(\sum\left|\hat{u}_{s}\right|^{2} s^{2 m}\right)^{k / m}=\|u\|_{0}^{1-k / m}\left(\left(\frac{\ell}{(2 \pi)}\right)^{2 m}\|u\|_{m}^{2}\right)^{k / m} .
\end{aligned}
$$

Доказательство неравенства (1.10) не является тривиальным. Интересующийся читатель может найти его в [4]. Там же найдено точное значение констант $C_{m k}$ в неравенстве (1.10):

$$
C_{m k}=\frac{K_{m-k}}{K_{m}^{1-k / m}}, \quad \text { где } K_{i}=\frac{4}{\pi}\left(1+\frac{(-1)^{i+1}}{3^{i+1}}+\frac{1}{5^{i+1}}+\frac{(-1)^{i+1}}{7^{i+1}}+\cdots\right) .
$$

Нам понадобится легко проверяемый факт, что

$$
\left(C_{m 1}\right)^{m} \leqslant 2 .
$$

Кроме этого, мы будем использовать неравенство Гальярдо-Ниренберга для периодических функций:

$$
\left|\frac{\partial^{\alpha} u}{\partial x^{\alpha}}\right|_{L_{2 m / \alpha}} \leqslant 4^{\alpha(m-\alpha)}|u|_{L_{\infty}}^{1-\alpha / m}\left|\frac{\partial^{m} u}{\partial x^{m}}\right|_{L_{2}}^{\alpha / m}
$$

где $\alpha$ - целое число между 0 и $m$ (см. [5, pp. 106-107]).

Символ $u^{(m)}$ обозначает $\partial^{m} u / \partial x^{m}$; аналогично $u_{x}=\partial u / \partial x, u_{x x}=\partial^{2} u / \partial x^{2}$ и $u_{t}=\partial u / \partial t$. Символ $L_{p}$, если не оговорено противное, обозначает пространство $L_{p}(S)$.

Автор благодарен проф. С. Б. Куксину за постановку задачи и внимание к работе.

\section{§2. Оценки сверху}

Обозначим через $u_{+}$и $u_{-}$соответственно максимум и минимум начальной

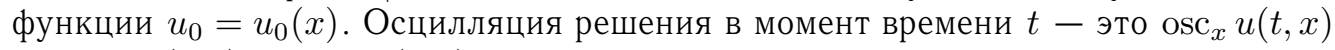
$=\max _{x} u(t, x)-\min _{x} u(t, x)$. Введем число

$$
D=\left.\frac{1}{2} \underset{x}{\operatorname{osc}} u\right|_{t=0}=\frac{u_{+}-u_{-}}{2} .
$$


Тогда в силу принципа максимума $u_{-} \leqslant u(t, x) \leqslant u_{+}$и

$$
\frac{1}{2} \underset{x}{\operatorname{osc}} u(t, x) \leqslant D \quad \text { для любого } t .
$$

Умножим уравнение (1.1) на $u$ и проинтегрируем по периоду:

$$
\int u u_{t} d x=-\delta \int u_{x}^{2} d x
$$

или

$$
\delta \int u_{x}^{2} d x=-\int u u_{t} d x \leqslant \frac{\operatorname{osc}_{x} u(t, x)}{2}\left|u_{t}\right|_{L_{1}} \leqslant\left. D\left|u_{t}\right|_{t=0}\right|_{L_{1}} .
$$

В последнем неравенстве мы воспользовались неравенством (2.2) и тем фактом, что величина $\left|u_{t}\right|_{L_{1}}=\int|(\partial u(t, x) / \partial t)| d x$ не возрастает по $t$ (см. лемму $\mathrm{A}$ в дополнении). Положим

$$
C_{1}=\sqrt{D \cdot\left(\int\left|\frac{d f\left(u_{0}\right)}{d x}\right| d x+\int\left|\frac{d^{2} u_{0}}{d x^{2}}\right| d x\right)} .
$$

Выражая $\left.u_{t}\right|_{t=0}$ из уравнения (1.1), находим, что

$$
\left\|u^{\delta}\right\|_{1} \leqslant C_{1} \delta^{-1 / 2}
$$

при всех $t \geqslant 0$ и $0<\delta<1$.

Дифференцируем уравнение (1.1) $m-1$ раз по переменной $x$, умножаем на $\partial^{m+1} u / \partial x^{m+1}$, интегрируем по периоду и результат запишем в таком виде:

$$
\frac{1}{2} \frac{d}{d t} \int\left(\frac{\partial^{m} u}{\partial x^{m}}\right)^{2} d x=-\delta \int\left(\frac{\partial^{m+1} u}{\partial x^{m+1}}\right)^{2} d x+\int \frac{\partial^{m+1} u}{\partial x^{m+1}} \frac{\partial^{m} f(u)}{\partial x^{m}} d x .
$$

Перед тем, как получить основные оценки этого параграфа, докажем вспомогательное техническое неравенство:

ЛЕммА. Для любого $m \geqslant 1$ имеет место неравенство

$$
\int\left|\frac{\partial^{m+1} u}{\partial x^{m+1}}\right|\left|\frac{\partial^{m} f(u)}{\partial x^{m}}\right| d x \leqslant B_{m}\|u\|_{m}\|u\|_{m+1},
$$

где

$$
B_{m}=Q_{m}|f(\cdot)|_{C^{m}\left[u_{-}, u_{+}\right]} \max \left(1,\left|u_{0}\right|_{L_{\infty}}^{m-1}\right),
$$

a $Q_{m}$ зависит лищь от $m$.

ДокАЗАТЕЛЬСтво. Методом математической индукции легко можно вывести следующую формулу:

$$
\frac{\partial^{m} f(u)}{\partial x^{m}}=\sum_{j=1}^{m} \frac{d^{j} f(u)}{d u^{j}}\left(\sum_{\substack{\alpha_{1}+\cdots+\alpha_{j}=m \\ 1 \leqslant \alpha_{1} \leqslant \cdots \leqslant \alpha_{j}}} A_{\alpha_{1}, \ldots, \alpha_{j}}^{j, m} u^{\left(\alpha_{1}\right)} u^{\left(\alpha_{2}\right)} \cdots u^{\left(\alpha_{j}\right)}\right),
$$


где $A_{\alpha_{1}, \ldots, \alpha_{j}}^{j, m}$ - некоторые неотрицательные целые числа. Следовательно,

$$
\begin{aligned}
& \int\left|\frac{\partial^{m+1} u}{\partial x^{m+1}}\right|\left|\frac{\partial^{m} f(u)}{\partial x^{m}}\right| d x \\
& \quad \leqslant \sum_{\substack { j=1 \\
\begin{subarray}{c}{\alpha_{1}+\cdots+\alpha_{j}=m \\
1 \leqslant \alpha_{1} \leqslant \ldots \leqslant \alpha_{j}{ j = 1 \\
\begin{subarray} { c } { \alpha _ { 1 } + \cdots + \alpha _ { j } = m \\
1 \leqslant \alpha _ { 1 } \leqslant \ldots \leqslant \alpha _ { j } } }\end{subarray}} A_{\alpha_{1}, \ldots, \alpha_{j}}^{j, m}\left|\frac{d^{j} f(u)}{d u^{j}}\right| \int\left|u^{(m+1)}\right|\left|u^{\left(\alpha_{1}\right)}\right| \cdots\left|u^{\left(\alpha_{j}\right)}\right| d x
\end{aligned}
$$

(применяем неравенство Гёльдера (заметим, что $\left.1 / 2+\alpha_{1} /(2 m)+\cdots+\alpha_{j} /(2 m)=1\right)$ )

$$
\leqslant \sum_{j=1}^{m} \sum_{\substack{\alpha_{1}+\cdots+\alpha_{j}=m \\ 1 \leqslant \alpha_{1} \leqslant \cdots \leqslant \alpha_{j}}} A_{\alpha_{1}, \ldots, \alpha_{j}}^{j, m}\left|\frac{d^{j} f(u)}{d u^{j}}\right|_{L_{\infty}}\left|u^{(m+1)}\right|_{L_{2}}\left|u^{\left(\alpha_{1}\right)}\right|_{L_{2 m / \alpha_{1}}} \cdots\left|u^{\left(\alpha_{j}\right)}\right|_{L_{2 m / \alpha_{j}}}
$$

(используем неравенство (1.12) (заметив, что $\left.\prod_{i=1}^{j} 4^{\alpha_{i}\left(m-\alpha_{i}\right)} \leqslant 4^{m^{2}}\right)$ )

$$
\begin{aligned}
& \leqslant \sum_{j=1}^{m} \sum_{\substack{\alpha_{1}+\cdots+\alpha_{j}=m \\
1 \leqslant \alpha_{1} \leqslant \cdots \leqslant \alpha_{j}}} A_{\alpha_{1}, \ldots, \alpha_{j}}^{j, m}\left|\frac{d^{j} f(u)}{d u^{j}}\right|_{L_{\infty}}\left|u^{(m+1)}\right|_{L_{2}} 4^{m^{2}}|u|_{L_{\infty}}^{j-1}\left|u^{(m)}\right|_{L_{2}} \\
& =\left(\sum_{j=1}^{m} \sum_{\substack{\alpha_{1}+\cdots+\alpha_{j}=m \\
1 \leqslant \alpha_{1} \leqslant \cdots \leqslant \alpha_{j}}} A_{\alpha_{1}, \ldots, \alpha_{j}}^{j, m} 4^{m^{2}}\left|\frac{d^{j} f(u)}{d u^{j}}\right|_{L_{\infty}}|u|_{L_{\infty}}^{j-1}\right)\|u\|_{m}\|u\|_{m+1} .
\end{aligned}
$$

Так как $|u|_{L_{\infty}} \leqslant\left|u_{0}\right|_{L_{\infty}}$ по принципу максимума, то лемма доказана.

Из (2.6) и (2.7) следует оценка

$$
\frac{1}{2} \frac{d}{d t}\|u\|_{m}^{2} \leqslant-\delta\|u\|_{m+1}^{2}+B_{m}\|u\|_{m}\|u\|_{m+1}=\|u\|_{m+1}\left(-\delta\|u\|_{m+1}+B_{m}\|u\|_{m}\right) .
$$

TЕOPЕмА. При $m \in \mathbb{N}$,

$$
\delta \leqslant \delta_{0}^{\prime}=B_{m}\left(\frac{C_{1}}{\sqrt{B_{m}}\left\|u_{0}\right\|_{m}}\right)^{1 /(m-1 / 2)}
$$

u при всех $t \geqslant 0$ имеет место неравенство

$$
\|u(t, \cdot)\|_{m} \leqslant B_{m}^{m-1} C_{1} \delta^{-m+1 / 2},
$$

где $C_{1}$ и $B_{m}$ определены в (2.4) и (2.8).

ДокАЗАТЕЛьСтво. Интерполяционное неравенство (1.9) запишем для производной:

$$
\|u\|_{m} \leqslant\|u\|_{1}^{1 / m}\|u\|_{m+1}^{(m-1) / m}
$$

и перепишем его в таком виде: $\|u\|_{m+1} \geqslant\|u\|_{m}\left(\|u\|_{m} /\|u\|_{1}\right)^{1 /(m-1)}$. Теперь из (2.9) с учетом последнего неравенства получаем

$$
\frac{1}{2} \frac{d}{d t}\|u\|_{m}^{2} \leqslant\|u\|_{m+1}\|u\|_{m}\left(-\delta\left(\frac{\|u\|_{m}}{\|u\|_{1}}\right)^{1 /(m-1)}+B_{m}\right) .
$$

То есть при $\|u\|_{m}>\left(B_{m} / \delta\right)^{m-1}\|u\|_{1}$ норма $\|u\|_{m}$ убывает. С учетом (2.5) заключаем, что

$$
\text { если }\|u(t, \cdot)\|_{m}>B_{m}^{m-1} C_{1} \delta^{-m+1 / 2}, \text { то норма }\|u\|_{m} \text { убывает. }
$$


Условие (2.10) означает, что неравенство (2.11) выполняется при $t=0$. А в силу импликации (2.13) норма $\|u\|_{m}$ не сможет превзойти правую часть этого неравенства.

\section{§3. Оценки снизу}

В этом параграфе мы воспользуемся явной формулой для слабого энтропийного решения $u^{0}(t, x)$ квазилинейного уравнения $\left(1.1^{\prime}\right)$, полученной Лаксом (см. [6]).

Пусть функция $g$ является преобразованием Лежандра выпуклой гладкой функции $f$. Пусть $y_{\min }=y_{\min }(t, x)$ обозначает точку глобального минимума вспомогательной функции $\psi_{t, x}(y)=\int_{0}^{y} u_{0}(\eta) d \eta+t \cdot g((x-y) / t)$. Эта точка однозначно определена при почти всех $t$ и $x$. Тогда $u^{0}(t, x)=g^{\prime}\left(\left(x-y_{\min }(t, x)\right) / t\right)$.

Пусть $M=\ell^{-1} \int u_{0} d x-$ среднее значение начальных данных. Тогда из явной формулы для решения уравнения $\left(1.1^{\prime}\right)$ в нашем ( $\ell$-периодическом) случае следует оценка

$$
\sup _{x}\left|u^{0}(t, x)-M\right| \leqslant \frac{\ell}{t \sigma} .
$$

Действительно, пусть $y_{1}$ - точка минимума функции $t \cdot g((x-y) / t)+M y$, т. е. $g^{\prime}\left(\left(x-y_{1}\right) / t\right)=M$. Поскольку функции $f^{\prime}$ и $g^{\prime}$ взаимно обратны, то $\left(x-y_{1}\right) / t=$ $f^{\prime}(M)$. Функция $\int_{0}^{y} u_{0}(\eta) d \eta-M y$ является $\ell$-периодической; поэтому $\left|y_{\min }-y_{1}\right|$ $<\ell$. Таким образом, $\left(x-y_{\min }\right) / t=f^{\prime}(M)+\kappa$, где $|\kappa| \leqslant \ell / t$. Теперь мы можем написать

$$
\left|u^{0}(t, x)-M\right|=\left|g^{\prime}\left(f^{\prime}(M)+\kappa\right)-g^{\prime}\left(f^{\prime}(M)\right)\right| \leqslant \sup \left|g^{\prime \prime}\right| \cdot|\kappa| \leqslant \frac{1}{\sigma} \frac{\ell}{t},
$$

что и утверждается в (3.1). Как следствие получаем, что $\int\left(u^{0}(t, x)-M\right)^{2} d x \leqslant$ $\ell^{3} /\left(t^{2} \sigma^{2}\right)$. Таким образом, в момент времени

$$
T=\frac{2 \ell^{3 / 2}}{\sigma\left|u_{0}(\cdot)-M\right|_{L_{2}}}
$$

справедлива оценка

$$
\int\left(u^{0}(T, x)-M\right)^{2} d x \leqslant \frac{1}{4}\left|u_{0}(\cdot)-M\right|_{L_{2}}^{2} .
$$

Далее для упрощения формул мы будем предполагать, что $M=0$. При этом мы не ограничиваем общности, так как можем сделать замену $u \rightarrow u-M$, $f(\cdot) \rightarrow f(\cdot+M)$.

TEOPEMA. Прu $m \in \mathbb{N} u$

$$
\delta<\delta_{0}^{\prime \prime}=\frac{1}{3072} \frac{\left|u_{0}\right|_{L_{2}}^{4}}{T\left|u_{0}\right|_{L_{\infty}}^{2}\left(\int\left|d u_{0} / d x\right| d x\right)^{2}}
$$

имеет место неравенство

$$
\left(\int_{0}^{T}\|u(t, \cdot)\|_{m}^{2} d t\right)^{1 / 2} \geqslant c_{m}\left(\frac{1}{\delta}\right)^{m-1 / 2} .
$$

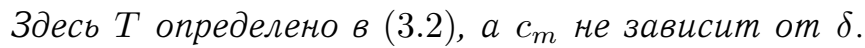

ДокАЗАТЕЛьство. Перепишем равенство (2.3) в таком виде:

$$
\delta \int\left(u_{x}^{\delta}(t, x)\right)^{2} d x=-\frac{1}{2} \frac{d}{d t} \int\left(u^{\delta}(t, x)\right)^{2} d x,
$$


и проинтегрируем его по $t$ от 0 до $T$ :

$$
\int_{0}^{T} \delta\left\|u^{\delta}\right\|_{1}^{2} d t=\frac{1}{2} \int\left(u^{\delta}(0, x)\right)^{2} d x-\frac{1}{2} \int\left(u^{\delta}(T, x)\right)^{2} d x .
$$

Неравенство (3.4) при $m=1$ будет доказано, как только мы покажем, что последний интеграл в (3.5) мал. Пусть $\delta<\delta_{0}^{\prime \prime}$; тогда, используя неравенство (Д.6) из дополнения, можем записать

$$
\begin{aligned}
\int\left(u^{\delta}(T, x)\right)^{2} d x-\int\left(u^{0}(T, x)\right)^{2} d x & \leqslant \int\left|u^{\delta}(T, x)-u^{0}(T, x)\right|\left|u^{\delta}(T, x)+u^{0}(T, x)\right| d x \\
& \leqslant \frac{4}{\sqrt{3}} \sqrt{T \delta} \int\left|\frac{d u_{0}}{d x}\right| d x \cdot 2\left|u_{0}\right|_{L_{\infty}} \leqslant \frac{1}{12}\left|u_{0}\right|_{L_{2}}^{2} .
\end{aligned}
$$

Теперь (3.5) и (3.3) вместе с последним неравенством дают

$$
\int_{0}^{T} \delta\left\|u^{\delta}\right\|_{1}^{2} d t \geqslant \frac{1}{2}\left|u_{0}\right|_{L_{2}}^{2}-\frac{1}{2}\left(\frac{1}{4}\left|u_{0}\right|_{L_{2}}^{2}+\frac{1}{12}\left|u_{0}\right|_{L_{2}}^{2}\right)=\frac{1}{3}\left|u_{0}\right|_{L_{2}}^{2},
$$

или

$$
\frac{1}{T} \int_{0}^{T}\left\|u^{\delta}\right\|_{1}^{2} d t \geqslant \frac{\left|u_{0}\right|_{L_{2}}^{2}}{3 T \delta}
$$

Тем самым доказано неравенство (3.4) при $m=1$ с константой $c_{1}=\left|u_{0}\right|_{L_{2}} / \sqrt{3 T}$. Из интерполяционного неравенства (1.10) с $k=1$ следует, что $\left|u_{x}\right|_{L_{\infty}} \leqslant$ $C_{m 1} D^{(m-1) / m}\left|u^{(m)}\right|_{L_{\infty}}^{1 / m}$, где $D$ определено в $(2.1)$ и использована формула $(2.2)$. Учитывая (1.11), имеем $\left|u^{(m)}\right|_{L_{\infty}} \geqslant\left(\left|u_{x}\right|_{L_{\infty}}\right)^{m} /\left(2 D^{m-1}\right)$. Используя неравенство (Д.4), мы можем записать $\left|\partial u^{\delta} / \partial x\right|_{L_{\infty}} \geqslant\left\|u^{\delta}\right\|_{1}^{2} /\left|d u_{0} / d x\right|_{L_{1}}$. Таким образом,

$$
\left|u^{(m)}\right|_{L_{\infty}} \geqslant \frac{\left\|u^{\delta}\right\|_{1}^{2 m}}{2 D^{m-1}\left|d u_{0} / d x\right|_{L_{1}}^{m}} .
$$

Лемма. Если v - периодическая функция, принимающая значение нуль, то

$$
|v|_{L_{\infty}}^{2} \leqslant|v|_{L_{2}}\left|v_{x}\right|_{L_{2}} .
$$

ДОКАЗАТЕЛЬСТВо. Для любого $у$ имеем

$v^{2}(y)=\int_{v^{-1}(0)}^{y} \frac{d}{d x} v^{2}(x) d x \leqslant \frac{1}{2} \int\left|\frac{d}{d x} v^{2}(x)\right| d x=\int|v(x)|\left|v^{\prime}(x)\right| d x \leqslant|v|_{L_{2}}\left|v_{x}\right|_{L_{2}}$.

Применяя предыдущую лемму к $v=\partial^{m} u / \partial x^{m}$ и используя неравенство $(2.12)$, получаем

$$
\left|u^{(m)}\right|_{L_{\infty}} \leqslant\|u\|_{m}^{1 / 2}\|u\|_{m+1}^{1 / 2} \leqslant\|u\|_{1}^{1 /(2 m)}\|u\|_{m+1}^{(2 m-1) /(2 m)} .
$$

Следовательно,

$$
\|u\|_{m+1} \geqslant\left(\frac{\left|u^{(m)}\right|_{L_{\infty}}^{2 m}}{\|u\|_{1}}\right)^{1 /(2 m-1)} \stackrel{(3.7)}{\geqslant} \frac{\|u\|_{1}^{2 m+1}}{\left(2 D^{m-1}\left|d u_{0} / d x\right|_{L_{1}}^{m}\right)^{2 m /(2 m-1)}} .
$$

Теперь возводим это неравенство в квадрат и, используя неравенство

$$
\frac{1}{T} \int_{0}^{T}\|u\|_{1}^{4 m+2} d t \geqslant\left(\frac{1}{T} \int_{0}^{T}\|u\|_{1}^{2} d t\right)^{2 m+1}
$$


усредняем по времени от 0 до $T$ :

$$
\begin{aligned}
\frac{1}{T} \int_{0}^{T}\|u\|_{m+1}^{2} d t & \geqslant \frac{\left(\frac{1}{T} \int_{0}^{T}\|u\|_{1}^{2} d t\right)^{2 m+1}}{\left(2 D^{m-1}\left|d u_{0} / d x\right|_{L_{1}}^{m}\right)^{4 m /(2 m-1)}} \\
& \geqslant \frac{\left(\left|u_{0}\right|_{L_{2}}^{2} /(3 T \delta)\right)^{2 m+1}}{\left(2 D^{m-1}\left|d u_{0} / d x\right|_{L_{1}}^{m}\right)^{4 m /(2 m-1)}} .
\end{aligned}
$$

Тем самым оценка (3.4) доказана при всех $m \in \mathbb{N}$.

Интересно сравнить неравенство (3.4) с $m=1$ с верхней границей для усредненной $L_{1}$-нормы, вытекающей из оценки (Д.4):

$$
\left\langle\int u^{\prime}(x)^{2} d x\right\rangle^{1 / 2} \geqslant c_{1} \delta^{-1 / 2}, \quad\left\langle\int\left|u^{\prime}(x)\right| d x\right\rangle \leqslant \text { Const . }
$$

Грубо говоря, эти неравенства означают, что график градиента $u^{\prime}(x)$ является кривой, у которой наблюдаются высокие и редкие всплески. Такое поведение тесно связано с феноменом перемежаемости в теории турбулентности (см. [8]).

\section{§4. Поведение коэффициентов Фурье}

В этом параграфе мы докажем оценки (1.6)-(1.8).

Коэффициенты Фурье функции, определенной на окружности $S$, - это коэффициенты ее разложения по ортонормированному базису, образованному экспонентами $e_{k}(\cdot)$, где

$$
e_{k}(x)=\frac{1}{\sqrt{\ell}} \exp \left(\frac{2 \pi i k x}{\ell}\right), \quad k \in \mathbb{Z} .
$$

Разлагая по этому базису, мы записываем функцию $u(x)$ в виде

$$
u(x)=\sum_{k=-\infty}^{+\infty} \hat{u}_{k} e_{k}(x), \quad \text { где } \hat{u}_{k}=\frac{1}{\sqrt{\ell}} \int u(x) \exp \left(\frac{-2 \pi i k x}{\ell}\right) d x
$$

и записываем ее $H^{m}$-норму Соболева следующим образом:

$$
\|u\|_{m}^{2}=\left(\frac{2 \pi}{\ell}\right)^{2 m} \sum_{k=-\infty}^{+\infty} k^{2 m}\left|\hat{u}_{k}\right|^{2} .
$$

Положив

мы имеем

$$
\hat{a}_{k}^{2}=\frac{1}{T} \int_{0}^{T}\left|\hat{u}_{k}(t)\right|^{2} d t
$$

$$
\frac{1}{T} \int_{0}^{T}\|u\|_{m}^{2} d t=\left(\frac{2 \pi}{\ell}\right)^{2 m} \sum_{k=-\infty}^{+\infty} k^{2 m} \hat{a}_{k}^{2}
$$

Сочетая теперь результаты $\$ \$ 2$ и 3 с этой формулой, получаем, что для достаточно малых $\delta$ (точнее, для $\left.\delta<\delta_{0}=\min \left(1, \delta_{0}^{\prime}, \delta_{0}^{\prime \prime}\right)\right)$ при $m \geqslant 1$ справедливы оценки

$$
\tilde{c}_{m} \delta^{-2 m+1}<\sum_{k=-\infty}^{+\infty} k^{2 m} \hat{a}_{k}^{2}<\widetilde{C}_{m} \delta^{-2 m+1}
$$


где $\tilde{c}_{m}=(\ell /(2 \pi))^{2 m} c_{m}^{2}$ и $\widetilde{C}_{m}=(\ell /(2 \pi))^{2 m} C_{m}^{2}$. Положим

$$
V=\frac{\ell}{4 \pi^{2}}\left|\frac{d u_{0}}{d x}\right|_{L_{1}}^{2}
$$

Тогда

$$
\left|\hat{u}_{k}\right|=\left|\frac{i \sqrt{\ell}}{2 \pi k} \int \frac{\partial u}{\partial x} \exp \left(\frac{-2 \pi i k x}{\ell}\right) d x\right| \leqslant \frac{\sqrt{\ell}}{2 \pi|k|}\left|\frac{\partial u}{\partial x}\right|_{L_{1}} \leqslant \frac{V^{1 / 2}}{|k|}
$$

В последнем неравенстве мы использовали оценку (Д.4) из дополнения. Таким образом, $\hat{a}_{k}^{2} \leqslant V / k^{2}$.

Для произвольных $s \geqslant 0$ и $\theta>0$ будем по-прежнему обозначать через $\hat{A}_{s, \theta}^{2}$ среднее значение коэффициентов $\hat{a}_{k}^{2}$ при $|k| \in\left(\delta^{-s+\theta} ; \delta^{-s-\theta}\right]$. Пусть выполнено условие $\delta^{\theta}<1 / 2$, которое обеспечивает непустоту множества индексов $k$, по которым происходит усреднение. Тогда из последнего неравенства (с использованием простого факта $\left.k^{-2}<\int_{k-1 / 2}^{k+1 / 2} x^{-2} d x\right)$ следует

$$
\hat{A}_{s, \theta}^{2}<\frac{V \int_{\left[\delta^{-s+\theta}\right]+1 / 2}^{\left[\delta^{-s-\theta}\right]+1 / 2} x^{-2} d x}{\left[\delta^{-s-\theta}\right]-\left[\delta^{-s+\theta}\right]}=\frac{V}{\left(\left[\delta^{-s-\theta}\right]+1 / 2\right)\left(\left[\delta^{-s+\theta}\right]+1 / 2\right)}<4 V \delta^{2 s} .
$$

Мы получили оценку (1.6).

Выведем оценку (1.7). Пусть $1<s-\theta<s<s+\theta, \delta^{\theta}<1 / 2$ и выполнено условие $(*)$; тогда

$\sum_{|k|>\delta^{-s+\theta}} \hat{a}_{k}^{2} \leqslant\left(\delta^{s-\theta}\right)^{2 m} \sum_{|k|>\delta^{-s+\theta}} k^{2 m} \hat{a}_{k}^{2} \leqslant\left(\delta^{s-\theta}\right)^{2 m} \sum_{k=-\infty}^{+\infty} k^{2 m} \hat{a}_{k}^{2} \leqslant \widetilde{C}_{m} \delta^{2 m(s-\theta-1)+1}$

и

$$
\hat{A}_{s, \theta}^{2} \leqslant \frac{\sum_{|k|>\delta^{-s+\theta}} \hat{a}_{k}^{2}}{2\left(\left[\delta^{-s-\theta}\right]-\left[\delta^{-s+\theta}\right]\right)} \leqslant \frac{\widetilde{C}_{m} \delta^{2 m(s-\theta-1)+1}}{\delta^{-s-\theta}}<\widetilde{C}_{m} \delta^{2+2 m(s-\theta-1)} .
$$

Оценка (1.7) доказана.

Найдем оценку снизу для среднего значения коэффициентов $\hat{a}_{k}^{2}$ при $|k| \in$ $\left(\delta^{\theta-1}, \delta^{-\theta-1}\right]$. Будем, как и в $\S 1$, обозначать это среднее через $\hat{A}_{1, \theta}^{2}$. Пусть выполнено условие $(*)$ и $\left(2 V+\widetilde{C}_{2}\right) \delta^{\theta} / \tilde{c}_{1}<1 / 2$. Тогда, так как

$$
\sum_{|k|>\delta^{-1-\theta / 2}} k^{2} \hat{a}_{k}^{2} \leqslant \delta^{2+\theta} \sum_{|k|>\delta^{-1-\theta / 2}} k^{4} \hat{a}_{k}^{2} \leqslant \widetilde{C}_{2} \delta^{-1+\theta}
$$

и

$$
\sum_{|k| \leqslant \delta^{-1+\theta}} k^{2} \hat{a}_{k}^{2} \leqslant 2 \sum_{0<k \leqslant \delta^{-1+\theta}} V \leqslant 2 V \delta^{-1+\theta}
$$


TO

$$
\begin{aligned}
& \sum_{\delta^{-1+\theta}<|k| \leqslant \delta^{-1-\theta}} \hat{a}_{k}^{2} \geqslant \delta^{2+\theta} \sum_{\delta^{-1+\theta}<|k| \leqslant \delta^{-1-\theta / 2}} k^{2} \hat{a}_{k}^{2} \\
&=\delta^{2+\theta}\left(\sum_{k=-\infty}^{+\infty} k^{2} \hat{a}_{k}^{2}-\sum_{|k| \leqslant \delta^{-1+\theta}} k^{2} \hat{a}_{k}^{2}-\sum_{|k|>\delta^{-1-\theta / 2}} k^{2} \hat{a}_{k}^{2}\right) \\
& \geqslant \tilde{c}_{1} \delta^{1+\theta}\left(1-\frac{2 V+\widetilde{C}_{2}}{\tilde{c}_{1}} \delta^{\theta}\right) \geqslant \frac{\tilde{c}_{1}}{2} \delta^{1+\theta}
\end{aligned}
$$

Таким образом,

$$
\hat{A}_{1, \theta}^{2} \geqslant \frac{\tilde{c}_{1} \delta^{1+\theta} / 2}{2 \delta^{-1-\theta}}=\frac{\tilde{c}_{1}}{4} \delta^{2+2 \theta},
$$

т. е. неравенство (1.8) доказано.

\section{Дополнение}

Пусть функция $u=u^{\delta}(t, x)$ удовлетворяет уравнению

$$
\frac{\partial}{\partial t} u+\frac{\partial f(u)}{\partial x}=\delta u_{x x}
$$

в цилиндре $[0,+\infty) \times S$ с начальным значением

$$
u^{\delta}(0, x)=u_{0}(x) \in C^{\infty}(S) .
$$

Здесь $f: \mathbb{R} \rightarrow \mathbb{R}-$ произвольная $C^{\infty}$-гладкая (не обязательно выпуклая) функция, $\delta>0$. Тогда (см. [1]) функция $u$ будет бесконечно гладкой при всех $t>0$.

Мы напомним, что если функция $v(t, x)$ удовлетворяет уравнению

$$
v_{t}+\psi(t, x) v_{x}=\delta v_{x x}
$$

где $\psi(t, x)$ есть $C^{\infty}$-гладкая функция, то для $v(t, x)$ имеет место принцип максимума: при $t_{1}<t_{2}$

$$
\inf v\left(t_{1}, \cdot\right) \leqslant \inf v\left(t_{2}, \cdot\right) \leqslant \sup v\left(t_{2}, \cdot\right) \leqslant \sup v\left(t_{1}, \cdot\right) .
$$

Пусть символ $\dot{u}$ обозначает либо функцию $\partial u / \partial t$, либо $\partial u / \partial x$ (два случая). Рассмотрим $L_{1}$-норму функции $\dot{u}$ по $x$ как функцию от $t,|\dot{u}|_{L_{1}}(t)=\int|\dot{u}(t, x)| d x$. В обоих случаях имеет место следующий факт:

ЛЕмма А. Функция $t \mapsto|\dot{u}|_{L_{1}}(t)$ не возрастает.

ДокАЗАТЕЛЬСтво. Функция $\dot{u}(t, x)$ удовлетворяет уравнению

$$
\dot{u}_{t}+\left(f^{\prime}(u) \dot{u}\right)_{x}=\delta \dot{u}_{x x} .
$$

Умножим его на произвольную гладкую в цилиндре $\left[T_{1}, T_{2}\right] \times S\left(T_{1}<T_{2}\right)$ функцию $\varphi(t, x)$ и проинтегрируем по частям:

$$
\left.\int \dot{u} \varphi\right|_{t=T_{2}} d x=\left.\int \dot{u} \varphi\right|_{t=T_{1}} d x+\int_{T_{1}}^{T_{2}} \int \dot{u}\left(\varphi_{t}+f^{\prime}(u) \varphi_{x}+\delta \varphi_{x x}\right) d x d t .
$$

Пусть теперь функция $\varphi(t, x)$ является решением задачи Коши для уравнения

$$
\varphi_{t}+f^{\prime}(u) \varphi_{x}+\delta \varphi_{x x}=0
$$


в цилиндре $\left[T_{1}, T_{2}\right] \times S$. Начальные данные для такого уравнения надо задавать на верхней крышке цилиндра, и мы полагаем

$$
\varphi\left(T_{2}, x\right)=\operatorname{sign}^{\varepsilon}\left(\dot{u}\left(T_{2}, x\right)\right),
$$

где функция $\operatorname{sign}^{\varepsilon}\left(\dot{u}\left(T_{2}, \cdot\right)\right)$ гладко аппроксимирует функцию $\operatorname{sign}\left(\dot{u}\left(T_{2}, \cdot\right)\right)$ с точностью до $\varepsilon$ в $L_{1}(S)$. Потребуем также выполнения неравенства

$$
\left|\operatorname{sign}^{\varepsilon}\left(\dot{u}\left(T_{2}, x\right)\right)\right| \leqslant 1 \text { для всех } x .
$$

Тогда в силу принципа максимума всюду в цилиндре $\left[T_{1}, T_{2}\right] \times S$ будет выполняться неравенство $|\varphi(t, x)| \leqslant 1$. Подставив $\varphi(t, x)$ в (Д.3), получим, что

$$
\int \dot{u}\left(T_{2}, x\right) \operatorname{sign}^{\varepsilon}\left(\dot{u}\left(T_{2}, x\right)\right) d x=\left.\int \dot{u} \varphi\right|_{t=T_{1}} d x \leqslant \int\left|\dot{u}\left(T_{1}, x\right)\right| d x .
$$

Устремив $\varepsilon$ к нулю, получаем

$$
\left.\int|\dot{u}|\right|_{t=T_{2}} d x \leqslant\left.\int|\dot{u}|\right|_{t=T_{1}} d x
$$

Отметим два простых следствия из доказанного факта. При любых $t \geqslant 0$ и $\delta>0$ имеет место неравенство

$$
\int\left|\frac{\partial u^{\delta}}{\partial x}(t, x)\right| d x \leqslant \int\left|\frac{d u_{0}}{d x}\right| d x .
$$

При $\delta \in(0,1)$ выполняется неравенство

$$
\int\left|u^{\delta}\left(t_{2}, x\right)-u^{\delta}\left(t_{1}, x\right)\right| d x \leqslant C\left|t_{2}-t_{1}\right|,
$$

причем константа $C$ не зависит от $\delta$; например, можно взять

$$
C=\int\left|\frac{d f\left(u_{0}\right)}{d x}\right| d x+\int\left|\frac{d^{2} u_{0}}{d x^{2}}\right| d x .
$$

Переходим к исследованию поведения решений задачи (Д.1), (Д.2) при $\delta \rightarrow 0$.

Нас интересует скорость сходимости к предельной функции, и мы получим следующий результат:

ТеоремА. Для любого $T \geqslant 0$ и любого $\delta \geqslant 0$ имеет место неравенство

$$
\int\left|u^{\delta}(T, x)-u^{0}(T, x)\right| d x \leqslant \frac{4}{\sqrt{3}} \sqrt{T \delta} \int\left|\frac{d u_{0}}{d x}(x)\right| d x .
$$

В дальнейшем мы будем опускать индекс $\delta$, а предельную функцию $u^{0}$ будем обозначать через $v$. Перед тем как доказывать эту теорему, установим ряд технических неравенств.

ЛЕммА 1. Для произвольного вещественного числа а и для любой $C^{2}$-гладкой неотрицательной функции $\psi(t, x)(t \in[0, T], x \in S)$ справедливо неравенство

$$
\begin{aligned}
& \int|u(T, x)-a| \psi(T, x) d x \leqslant \int|u(0, x)-a| \psi(0, x) d x \\
& +\int_{0}^{T} \int F(u(t, x), a) \psi_{x}(t, x)+|u(t, x)-a|\left(\psi_{t}(t, x)+\delta \psi_{x x}(t, x)\right) d x d t,
\end{aligned}
$$

где $F(u, a)=\operatorname{sign}(u-a)(f(u)-f(a))$. 
ДокАзАТЕЛЬСтво. Умножаем уравнение (Д.1) на $\Phi_{n}^{\prime}(u) \psi(t, x)$, где $\Phi_{n}(\cdot)-$ произвольная $C^{2}$-гладкая выпуклая $\left(\Phi_{n}^{\prime \prime}(\cdot) \geqslant 0\right)$ функция. Затем интегрируем по цилиндру $[0, T] \times S$ :

$$
\int_{0}^{T} \int \Phi_{n}^{\prime}(u) u_{t} \psi d x d t+\int_{0}^{T} \int \Phi_{n}^{\prime}(u) f^{\prime}(u) u_{x} \psi d x d t=\delta \int_{0}^{T} \int u_{x x} \Phi_{n}^{\prime}(u) \psi d x d t .
$$

Замечая, что $\Phi_{n}^{\prime}(u) f^{\prime}(u) u_{x}=(\partial / \partial x) \int_{0}^{u} \Phi_{n}^{\prime}(\xi) f^{\prime}(\xi) d \xi$ и интегрируя по частям, получаем

$$
\begin{aligned}
&\left.\int \Phi_{n}(u) \psi\right|_{t=T} d x-\left.\int \Phi_{n}(u) \psi\right|_{t=0} d x \\
&-\int_{0}^{T} \int \Phi_{n}(u) \psi_{t} d x d t-\int_{0}^{T} \int\left(\int_{0}^{u} \Phi_{n}^{\prime}(\xi) f^{\prime}(\xi) d \xi\right) \psi_{x} d x d t \\
& \quad=-\delta \int_{0}^{T} \int u_{x}^{2} \Phi_{n}^{\prime \prime}(u) \psi d x d t-\delta \int_{0}^{T} \int \Phi_{n}^{\prime}(u) u_{x} \psi_{x} d x d t,
\end{aligned}
$$

или

$$
\begin{aligned}
\left.\int \Phi_{n}(u) \psi\right|_{t=T} d x \leqslant \int & \left.\Phi_{n}(u) \psi\right|_{t=0} d x+\int_{0}^{T} \int\left(\int_{0}^{u} \Phi_{n}^{\prime}(\xi) f^{\prime}(\xi) d \xi\right) \psi_{x} d x d t \\
& +\int_{0}^{T} \int \Phi_{n}(u)\left(\psi_{t}+\delta \psi_{x x}\right) d x d t .
\end{aligned}
$$

Пусть теперь функции $\Phi_{n}(\cdot)$ аппроксимируют функцию $|\cdot-a|$ в равномерной метрике, причем

$$
\frac{d \Phi_{n}(u)}{d u} \rightarrow \frac{d}{d u}|u-a|=\left\{\begin{aligned}
1 & \text { при } u>a, \\
-1 & \text { при } u<a,
\end{aligned}\right.
$$

в $L_{1}\left(u_{-}, u_{+}\right)$, где $u_{-}=\min u_{0}, u_{+}=\max u_{0}$. Переходя к пределу, получаем (Д.7).

Заметим, что из принципа максимума для функций $u^{\delta}$ и условия (Д.4) следует, что семейство

$$
\left\{u^{\delta}(t, \cdot)\right\}_{\delta \in(0,1) ; t \in[0, T]}
$$

равномерно ограниченно и имеет равномерно ограниченную вариацию. Поэтому из всякого бесконечного подмножества этих функций по второй теореме Хелли (см. [7]) можно выбрать сходящуюся в каждой точке из $S$ последовательность. По теореме Лебега о предельном переходе под знаком интеграла она будет сходится в $L_{1}(S)$. Это означает, что семейство $(\star)$ предкомпактно в $L_{1}(S)$. В силу этого и согласно условию (Д.5) по теореме Арцела-Асколи семейство функций $\left\{u^{\delta}(t, x)\right\}_{\delta \in(0,1)}$ предкомпактно по норме пространства $C\left([0, T] ; L_{1}(S)\right)$. Отсюда заключаем, что найдется элемент $v \in C\left([0, T] ; L_{1}(S)\right)$ и последовательность $\delta_{k} \rightarrow 0(k \rightarrow \infty)$, такие, что $u^{\delta_{k}} \rightarrow v$ по норме пространства $C\left([0, T] ; L_{1}(S)\right)$.

Заметим, что из этой сходимости и принципа максимума для $u^{\delta_{k}}$ следует, что при каждом $t \in[0, T]$

$$
u_{-} \leqslant \operatorname{essinf} v(t, \cdot) \leqslant \operatorname{ess} \sup v(t, \cdot) \leqslant u_{+},
$$


так как иначе величина $\left|u^{\delta_{k}}(t, \cdot)-v(t, \cdot)\right|_{L_{1}}$ не стремилась бы к нулю. Для следующего предельного перехода воспользуемся неравенствами

$$
|| u(t, x)-a|-| v(t, x)-a|| \leqslant|u(t, x)-v(t, x)|
$$

и

$$
|F(u(t, x), a)-F(v(t, x), a)| \leqslant \max _{\left[u_{-}, u_{+}\right]}\left|f^{\prime}(\cdot)\right||u(t, x)-v(t, x)| \quad \text { при п.в. }(t, x) .
$$

(Первое неравенство - это просто неравенство треугольника, а для доказательства второго нужно рассмотреть два случая: a) $a$ находится между $u$ и $v$; b) $u$ и $v$ лежат по одну сторону от $a$. При каждом $t$ второе неравенство справедливо при почти всех $x$.)

Переходим к пределу в неравенстве (Д.7) по указанной подпоследовательности $\delta_{k} \rightarrow 0$ (при фиксированной функции $\psi(t, x)$ и при фиксированных начальных данных $\left.u_{0}\right)$ :

$$
\begin{aligned}
\int|v(T, \tilde{x})-b| \tilde{\psi} & (T, \tilde{x}) d \tilde{x} \leqslant \int|v(0, \tilde{x})-b| \tilde{\psi}(0, \tilde{x}) d \tilde{x} \\
& +\int_{0}^{T} \int\left(F(v(\tilde{t}, \tilde{x}), b) \tilde{\psi}_{\tilde{x}}(\tilde{t}, \tilde{x})+|v(\tilde{t}, \tilde{x})-b| \tilde{\psi}_{\tilde{t}}(\tilde{t}, \tilde{x})\right) d \tilde{x} d \tilde{t}
\end{aligned}
$$

Мы специально сделали замены $a \mapsto b$ и $t, x, \psi \mapsto \tilde{t}, \tilde{x}, \tilde{\psi}$.

Лемма 2. Пусть $\Omega: S \rightarrow \mathbb{R}-$ произвольная неотрицательная $C^{2}$-гладкая функция на окружности $S$. Тогда

$$
\begin{aligned}
\iint|u(T, x)-v(T, \tilde{x})| \Omega(x-\tilde{x}) & d x d \tilde{x} \leqslant \iint|u(0, x)-v(0, \tilde{x})| \Omega(x-\tilde{x}) d \tilde{x} d x \\
& +\delta \int_{0}^{T} \iint|u(t, x)-v(t, \tilde{x})| \Omega^{\prime \prime}(x-\tilde{x}) d x d \tilde{x} d t .
\end{aligned}
$$

ДоКАЗАТЕЛЬство. Пусть $\omega_{\varepsilon}: \mathbb{R} \rightarrow \mathbb{R}-$ дважды гладкая четная неотрицательная функция, равная нулю вне $\varepsilon$-окрестности нуля $\left(\omega_{\varepsilon}(z) \equiv 0\right.$ при $\left.|z| \geqslant \varepsilon\right)$, причем

$$
\int_{-\infty}^{+\infty} \omega_{\varepsilon}(z) d z=1 .
$$

Заметим, что функция $\hat{\psi}(t, \tilde{t}, x, \tilde{x})=\omega_{\varepsilon}(t-\tilde{t}) \Omega(x-\tilde{x})$ удовлетворяет условиям $\partial \hat{\psi} / \partial t=-\partial \hat{\psi} / \partial \tilde{t}$ и $\partial \hat{\psi} / \partial x=-\partial \hat{\psi} / \partial \tilde{x}$. Подставим в $($ Д.7) $a=v(\tilde{t}, \tilde{x})$ и $\psi(t, x)=$ $\hat{\psi}(t, \tilde{t}, x, \tilde{x})$ и проинтегрируем по $(\tilde{t}, \tilde{x}) \in[0, T] \times S$. Кроме этого, подставим в неравенство (Д.8) $b=u(t, x)$ и $\tilde{\psi}(\tilde{t}, \tilde{x})=\hat{\psi}(t, \tilde{t}, x, \tilde{x})$ и проинтегрируем по $(t, x) \in$ $[0, T] \times S$. Затем сложим два полученных неравенства:

$$
\begin{aligned}
\int_{0}^{T} \iint \mid & u(T, x)-v(\tilde{t}, \tilde{x}) \mid \omega_{\varepsilon}(T-\tilde{t}) \Omega(x-\tilde{x}) d x d \tilde{x} d \tilde{t} \\
& +\int_{0}^{T} \iint|v(T, \tilde{x})-u(t, x)| \omega_{\varepsilon}(t-T) \Omega(x-\tilde{x}) d \tilde{x} d x d t
\end{aligned}
$$




$$
\begin{aligned}
& \leqslant \int_{0}^{T} \iint|u(0, x)-v(\tilde{t}, \tilde{x})| \omega_{\varepsilon}(-\tilde{t}) \Omega(x-\tilde{x}) d x d \tilde{x} d \tilde{t} \\
& \quad+\int_{0}^{T} \iint|v(0, \tilde{x})-u(t, x)| \omega_{\varepsilon}(t) \Omega(x-\tilde{x}) d \tilde{x} d x d t \\
& \quad+\delta \int_{0}^{T} \int_{0}^{T} \iint|u(t, x)-v(\tilde{t}, \tilde{x})| \omega_{\varepsilon}(t-\tilde{t}) \frac{\partial^{2}}{\partial x^{2}} \Omega(x-\tilde{x}) d x d \tilde{x} d t d \tilde{t} .
\end{aligned}
$$

Переходя к пределу при $\varepsilon \rightarrow 0$, получаем утверждение леммы.

Лемма 3. Пусть $\Psi: \mathbb{R} \rightarrow \mathbb{R}-$ неотрицательная $C^{2}$-гладкая функизия $c$ компактным носителем. Тогда

$$
\begin{array}{r}
\int_{-\infty}^{+\infty} \int|u(T, x+y)-v(T, x)| \Psi(y) d x d y \leqslant \int_{-\infty}^{+\infty} \int|u(0, x+y)-v(0, x)| \Psi(y) d x d y \\
+\delta \int_{0}^{T} \int_{-\infty}^{+\infty} \int|u(t, x+y)-v(t, x)| \Psi^{\prime \prime}(y) d x d y d t . \quad \text { (Д.9) }
\end{array}
$$

ДокАЗАТЕЛЬСтво. Применим предыдущую лемму с $\Omega(\cdot)=\sum_{k=-\infty}^{+\infty} \Psi(\cdot+k \ell)$, заменив координаты $x-\tilde{x}$ на $y$ и $\tilde{x}$ на $x$.

Для получения необходимых следствий из (Д.9) нам потребуется воспользоваться неравенством треугольника в следующей форме:

$$
|u(t, x+y)-v(t, x)|=|u(t, x)-v(t, x)|+w(t, x, y),
$$

где $|w(t, x, y)| \leqslant|u(t, x+y)-u(t, x)|$.

Учитывая, что $u(0, x) \equiv v(0, x)$ и $\int_{-\infty}^{+\infty} \Psi^{\prime \prime}(y) d y=0$, получаем

$$
\begin{aligned}
\int|u(T, x)-v(T, x)| & d x \int_{-\infty}^{+\infty} \Psi(y) d y \leqslant \int_{-\infty}^{+\infty} \int|u(T, x)-u(T, x+y)| d x \Psi(y) d y \\
& +\int_{-\infty}^{+\infty} \int|u(0, x)-u(0, x+y)| d x \Psi(y) d y \\
& +\delta \int_{0}^{T} \int_{-\infty}^{+\infty} \int|u(t, x)-u(t, x+y)| d x\left|\Psi^{\prime \prime}(y)\right| d y d t . \quad \text { (Д.10) }
\end{aligned}
$$

Далее будем предполагать, что $\int_{-\infty}^{+\infty} \Psi(y) d y=1$. Воспользовавшись неравенствами

$$
\begin{aligned}
& \int|u(t, x)-u(t, x+y)| d x=\int\left|\int_{0}^{y} \frac{\partial u}{\partial x}(t, x+\xi) d \xi\right| d x \\
& \leqslant\left|\int_{0}^{y} \int\right| \frac{\partial u}{\partial x}(t, x+\xi)|d x d \xi|=|y| \int\left|\frac{\partial u}{\partial x}(t, x)\right| d x \stackrel{\text { (Д.4) }}{\leqslant}|y| \int\left|\frac{d u_{0}}{d x}\right| d x,
\end{aligned}
$$

перепишем (Д.10) в виде

$\int|u(T, x)-v(T, x)| d x \leqslant\left(2 \int_{-\infty}^{+\infty}|y| \Psi(y) d y+T \delta \int_{-\infty}^{+\infty}|y|\left|\Psi^{\prime \prime}(y)\right| d y\right) \int\left|\frac{d u_{0}}{d x}\right| d x$. 
Пусть теперь функции $\Psi(y)$ аппроксимируют функцию $\max ((1-|y| / \varepsilon) / \varepsilon, 0)$. Тогда выражение в скобках в (Д.11) будет стремится к

$$
2 \int_{-\varepsilon}^{\varepsilon}|y| \frac{1}{\varepsilon}\left(1-\frac{|y|}{\varepsilon}\right) d y+2 \delta T \frac{1}{\varepsilon}=\frac{2}{3}\left(\varepsilon+\frac{3 T \delta}{\varepsilon}\right) .
$$

Минимизируя по $\varepsilon$, получаем оценку (Д.6). Теорема доказана.

\section{ЛИТЕРАТУРА}

1. Ладьженская О. А., Солонников В. А., Уральцева Н. Н. Линейные и квазилинейные уравнения параболического типа. Наука, М., 1967.

2. Кружков $C$. H. Квазилинейные уравнения первого порядка со многими независимыми переменными. Матем. сб., 81 (123), № 2, 228-255 (1970).

3. Кузнецов H. Н. Точность некоторых приближенных методов расчета слабых решений квазилинейного уравнения первого порядка. Журнал выч. матем. и матем. физ., 16, № 6, 1489-1502 (1976).

4. Колмогоров A. H. О неравенствах между верхними гранями последовательных производных произвольной функции на бесконечном интервале. В кн.: Избранные труды. Математика и механика. Наука, М., 1985, с. 252-263.

5. Hörmander L. Lectures on nonlinear hyperbolic differential equations. Springer-Verlag, Berlin, 1997.

6. Lax P. D. Hyperbolic systems of conservation laws II. Comm. Pure Appl. Math., 10, 537-566 (1957).

7. Колмогоров A. Н., Фомин C. В. Элементы теории функций и функционального анализа. Наука, М., 1989.

8. Фрищ У. Турбулентность. Наследие А. Н. Колмогорова. Библиотека математика, вып. 4, Фазис, М., 1998.

9. Kuksin S. Spectral Properties of Solutions for Nonlinear PDE's in the Turbulent Regime. Geom. Funct. Anal., 9, 141-184 (1999).

10. E Weinan, Khanin K., Mazel A., Sinai Ya. Invariant measures for Burgers equation with stochastic forcing. Ann. of Math., 151, No. 3, 877-960 (2000).

Московский государственный университет, механико-математический факультет,

Поступило в редакцию Department of Mathematics,

Heriot-Watt University, Edinburgh 